\title{
Isolated tension pneumoperitoneum following endobronchial ultrasound-guided transbronchial needle aspiration complicated by cardiac peri-arrest: $\mathrm{A}$ case report
}

\author{
Piergiorgio Muriana, Angelo Carretta, Paola Ciriaco, Francesca Rossetti, Giampiero Negri \\ Department of Thoracic Surgery, San Raffaele Scientific Institute, Milan, Italy
}

\begin{abstract}
Transbronchial needle aspiration under endobronchial ultrasound guidance (EBUS-TBNA) is recommended for the diagnosis and staging of lung cancer. Major complications following EBUS-TBNA are uncommon. We report a case of isolated tension pneumoperitoneum following EBUS-TBNA under deep sedation in an 80-year-old male patient affected by a right hilar mass suspicious for lung cancer. At the end of the procedure, the patient suddenly manifested desaturation, severe bradycardia and hypotension, and abdominal distension. After resuscitation, in the suspect of intraperitoneal free air, needle decompression led to respiratory and hemodynamic improvement. A total body CT-scan showed isolated pneumoperitoneum without signs of gastrointestinal perforation, confirmed by a subsequent upper digestive contrast study. The patient recovered well without the need of surgical exploration. Isolated tension pneumoperitoneum can be an exceptional complication of EBUS-TBNA; a conservative treatment can avoid unnecessary surgery in the absence of peritonism.
\end{abstract}

Corresponding author: Piergiorgio Muriana, Department of Thoracic Surgery, San Raffaele Scientific Institute, Via Olgettina 60, 20132 Milan, Italy. Tel. +39.2.26437139 - Fax: +39.2.26437147.

E-mail: muriana.piergiorgio@hsr.it

Key words: EBUS; sedation; pneumoperitoneum; complication; conservative treatment.

Contributions: PM, data acquisition, manuscript drafting; AC, PC, FR, contribution to data acquisition and analysis, literature review; GN, contribution to manuscript writing and review. All the Authors approved the final version of the manuscript.

Conflict of interest: The Authors declare no conflict of interest.

Received for publication: 25 September 2018

Accepted for publication: 3 December 2018

(C) Copyright P. Muriana et al., 2018

Tipografia PI-ME Editrice, Italy

Monaldi Archives for Chest Disease 2018; 88:999

doi: 10.4081/monaldi.2018.999

This article is distributed under the terms of the Creative Commons Attribution Noncommercial License (by-nc 4.0) which permits any noncommercial use, distribution, and reproduction in any medium, provided the original author(s) and source are credited.

\section{Introduction}

Transbronchial needle aspiration under endobronchial ultrasound guidance (EBUS-TBNA) is considered the technique of choice for the diagnosis and staging of non-small cell lung cancer (NSCLC) [1].

Among the factors that may influence the success of the procedure, the use of sedation during EBUS-TBNA is associated with several advantages both for the operator and the patient. Some Authors [2,3] reported that the use of minimal sedation might allow higher levels of sampling adequacy, patient satisfaction, and cost reduction. However, the current guidelines of the American College of Chest Physicians (ACCP) [1] suggest the use of moderate to deep sedation to achieve better results in terms of diagnostic yield and procedure tolerance. The overall reported rate of major complications during EBUS-TBNA is around 0.15\% [4], and most of the minor complications resolve spontaneously.

Herein, we report a unique case of isolated tension pneumoperitoneum following EBUS-TBNA under deep sedation.

\section{Case Report}

An 80-year-old male patient was admitted to our Hospital for a parahilar mass of the right upper pulmonary lobe, enlarged ipsilateral mediastinal lymphnodes, and bilateral basal pleural effusions (Figure 1).

His past medical history was positive for prostatic cancer, hypertensive cardiopathy with normal left ventricular function, and insulin-dependent diabetes complicated by chronic renal failure. He had undergone kidney transplantation 11 years before. His allergic anamnesis was silent. In order to obtain a differential diagnosis of the pulmonary lesion and mediastinal staging, the patient was referred to our Bronchoscopy service and EBUS was scheduled.

The procedure was carried under deep sedation (propofol $430 \mathrm{mg}$ ) with anesthesiologic surveillance. Additional topical anesthesia was provided by administration of lidocaine $2 \%$. Oxygen was delivered through a nasopharyngeal catheter (NPC) with $15 \mathrm{~L} / \mathrm{min}$ flow rate. Bronchoscopic exploration showed an enlargement of the carina between the right upper lobe bronchus and the bronchus intermedius. Tissue samplings of the pulmonary mass and of ipsilateral paratracheal enlarged lymphnodes were obtained by means of EBUS-TBNA. Histopathology was positive for pulmonary adenocarcinoma. The entire procedure lasted $36 \mathrm{~min}$ overall.

In the few minutes following the procedure, the patient recovered well from sedation, and was awake with normal vital parameters. However, a few minutes later, he developed severe desaturation, edema of the tongue, and bronchospasm. As mask ventilation was ineffective, the anesthetist proceeded with endotracheal intubation. An initial esophageal misplacement of the tube was rapidly recognized and the endotracheal tube was correctly positioned. Bronchoscopy showed no lesions of the airway. In the suspicion of an allergic reaction, steroid, chlor- 
phenamine, salbutamol, and adrenaline $0.15 \mathrm{mg}$ were administered; moreover, mechanical ventilation was initiated following curarization.

A progressively worsening abdominal distension, severe bradycardia, and arterial hypotension were then observed. Nasogastric intubation produced no relevant changes. Hemodynamic instability with severe bradycardia was temporarily controlled by supplemental atropine $2 \mathrm{mg}$. An abdomen ultrasound showed an acoustic barrier indicative of a massive pneumoperitoneum. Bilateral pleural anterior sliding excluded the concurrent presence of major pneumothorax. Emergency decompression was achieved by positioning two large-bore cannulas bilaterally in the periumbilical region. Following needle decompression, hemodynamic and respiratory distress significantly improved.

The patient was transferred to the Radiology Department for a totalbody CT-scan, which confirmed the presence of a large anterior pneumoperitoneum with some small bubbles around the gastric body, but without fluid collections (Figure 2). Neither pneumothorax nor pneumomediastinum were observed.

A conservative treatment was chosen due to significant improvement of hemodynamic and ventilatory parameters, and the absence of reliable signs of significant esophageal or gastrointestinal perforation. The patient was transferred to the intensive care unit for respiratory weaning and was extubated $24 \mathrm{~h}$ later. No signs of peritonism emerged during the observation period.

An upper digestive CT-scan with Gastrografin did not show contrast leak (Figure 3); the subsequent clinical course was uneventful. The patient was finally discharged from the hospital 13 days later.

A specific consent for the publication of the case was acquired from the patient.

\section{Discussion}

A few cases of pneumoperitoneum have been described in the literature so far, in which no visceral lesion is identified at exploratory laparotomy. This condition is known as non-surgical pneumoperitoneum (NSP) [5].

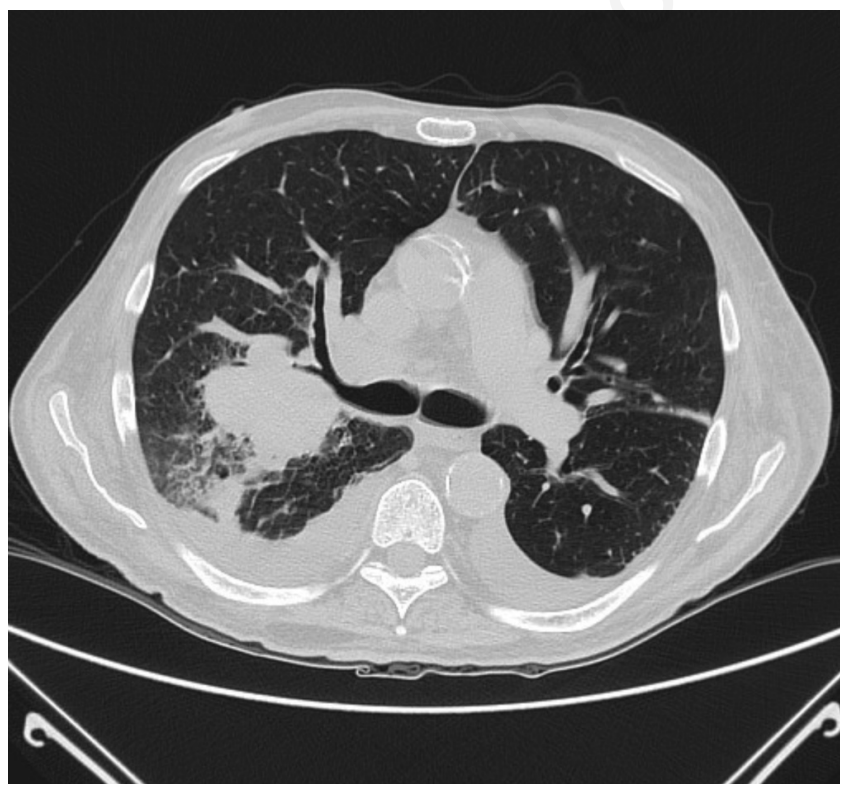

Figure 1. Admission axial chest CT-scan showing a $6 \mathrm{~cm}$-diameter mass in the right upper pulmonary lobe.
NSP causes can be classified as abdominal, thoracic, gynecologic, or idiopathic. The overall prevalence of thoracic etiologies of NSP is very low, and most of the cases are found in mechanically-ventilated newborns [5]. While pneumothorax and pneumomediastinum are known as rare complications of EBUS-TBNA [4], pneumoperitoneum following EBUS-TBNA under sedation has never been reported before in the literature. Since 2003, we performed a total of 1022 TBNA under sedation before the present case. Despite the very low incidence of such complication, all the clinicians involved in EBUS-TBNA (endoscopist, anesthetist, and nurses) should be aware of its existence. In fact, after the development of a tension pneumoperitoneum, the patient may show dramatic hemodynamic and respiratory failure in the range of few minutes, as happened in the case we observed. In such emergent clinical scenario, the application of appropriate diagnostic and therapeutic procedures is therefore of paramount importance.

The possibility of a pneumoperitoneum complicating a pneumothorax and/or a pneumomediastinum was excluded by the absence of endobronchial lesions at post-intubation bronchoscopy and negative chest CT-scan. Therefore, we evaluated two different mechanisms which may have led to the development of pneumoperitoneum.

First, pneumoperitoneum may possibly have originated from a visceral lesion caused by the oxygen flux through the NPC. Oxygen desaturation during bronchoscopy under deep sedation is a common event and up to $30 \%$ of patients show hypoxemia when low-flow rate oxygen is supplemented by means of nasal cannula because of ineffective nasal breathing and upper airway obstruction following sedation [6]. The use of NPC for oxygen delivery during flexible bronchoscopy in selected patients may reduce the incidence of hypoxic events [7]. In our experience, we observed that high-flow rate oxygen administration (up to $15 \mathrm{~L} / \mathrm{min}$ ) through NPC in sedated patients is useful to maintain a mild positive pressure contrasting airway obstruction and to deliver a high inspiratory oxygen fraction, which significantly reduces the incidence of desaturation events during the procedure.

It has been described [8] that some patients undergoing fiberoptic bronchoscopy under sedation and oxygen delivery by means of NPC experienced the development of a pneumoperitoneum secondary to gastric

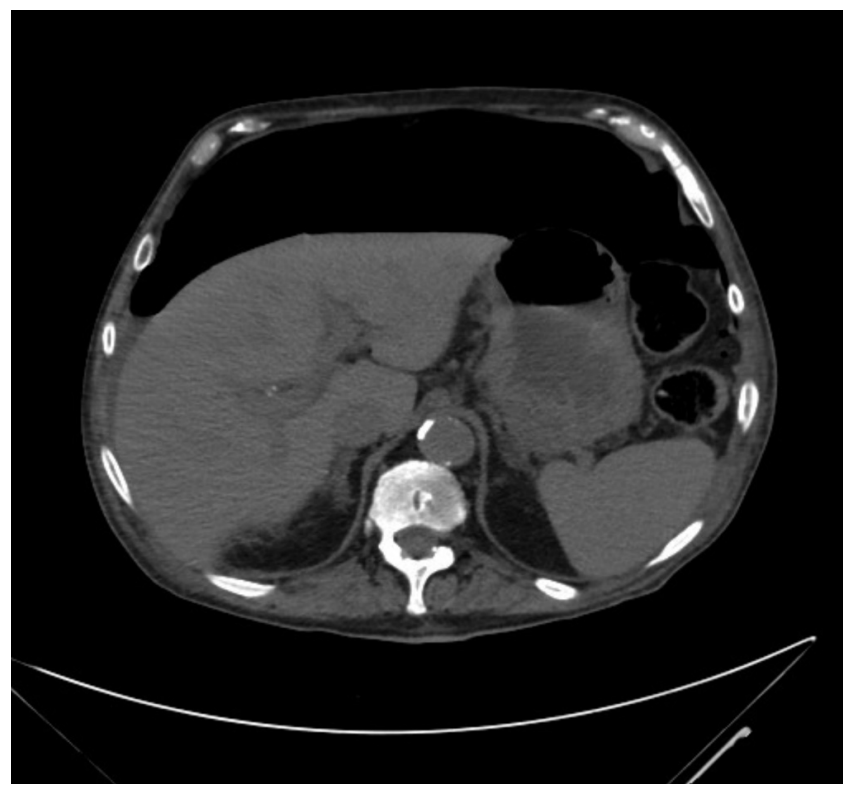

Figure 2. Abdominal CT-scan images taken after needle decompression demonstrating the presence of a large anterior pneumoperitoneum without fluid collections. 
rupture as result of the combination of factors, such as the reduction of the pharyngeal muscle tone induced by anesthetics, a passage of air in the stomach with one-way valve mechanism, and eventually NPC dislocation in the digestive tract. However, this hypothesis was not fully confirmed, considering the absence of upper digestive tract perforations at the contrast study. In addition, in our practice, we always start the procedure only after a check of the correct placement of the NPC.

The second mechanism hypothesized is an NSP caused by intubation or mechanical ventilation. Unrecognized esophageal intubation is a possible complication of emergency endotracheal tube insertion even in experienced hands [9]. In our case, nevertheless, esophageal intubation was promptly detected, and the patient was not ventilated until the correct position of the tube in the airway had been confirmed by bronchoscopy.

Over 100 cases of NSP secondary to mechanical ventilation have been previously reported [10]. In these patients, air passed into the peritoneal cavity through pleural, or diaphragmatic defects, or through the mediastinum and retroperitoneum [5,10]. However, differently from our present case, the simultaneous presence of pneumothorax and/or pneumomediastinum is a constant finding at diagnosis [10]. Although the pathogenesis of pneumoperitoneum could not be clearly demonstrated, both mechanisms may have had a role in the development of this infrequent complication.

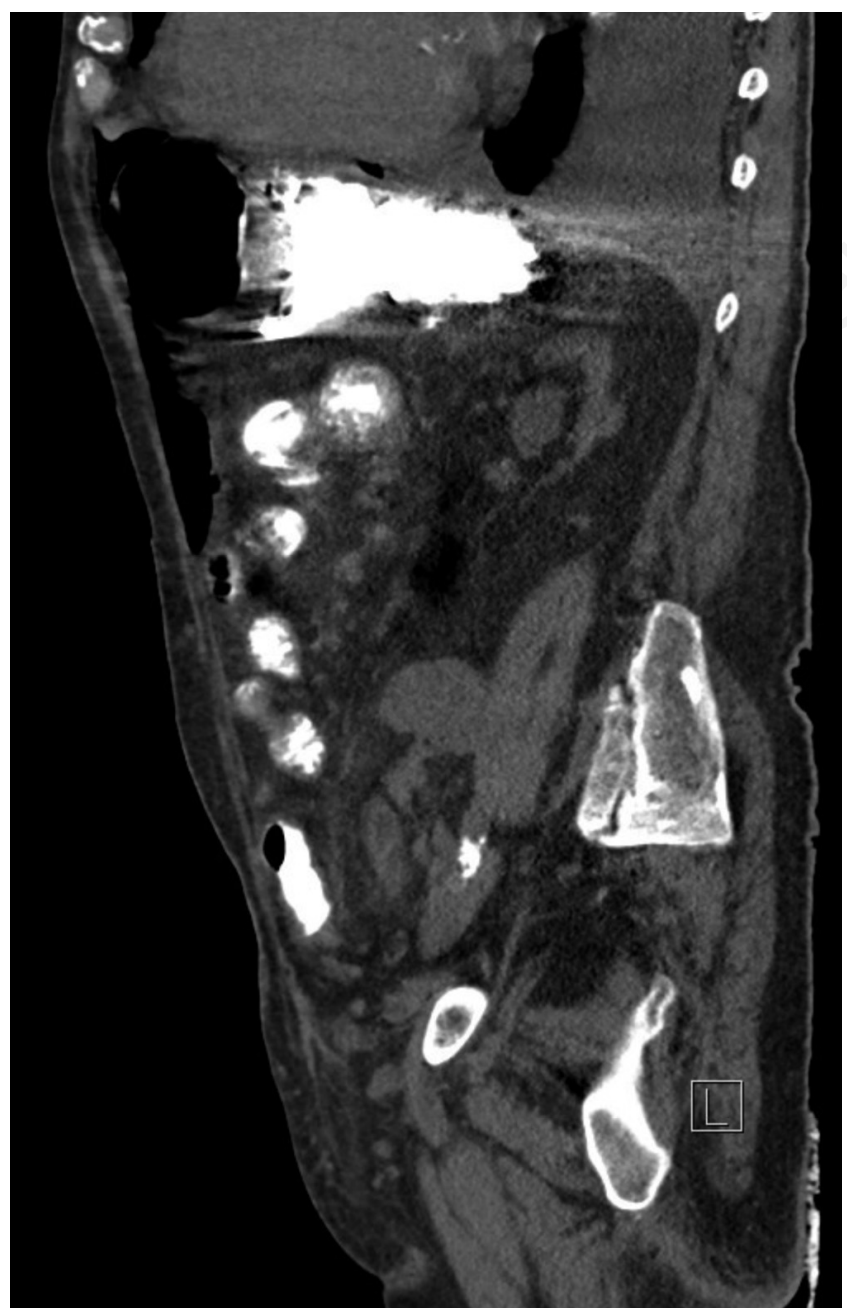

Figure 3. After extubation, the patient underwent a gastrographic upper digestive study, which resulted negative for contrast leak.
Hence, isolated iatrogenic tension NSP is an exceptional finding [11], and this is the first case described following EBUS-TBNA. The passage of air in the peritoneal cavity was most likely due to a gastric or bowel microperforation, similarly to what happens during endoscopic submucosal dissection of gastric tumors [12]. Such lesions are not visible at endoscopic view, but their presence can be indirectly confirmed by the observation of intraperitoneal air at CT-scan.

Although the consequences of tension NSP causing hemodynamic instability can be serious, a conservative approach is preferable, and can be considered as definitive treatment in absence of peritoneal irritation [10]. In fact, needle decompression or percutaneous drainage are effective methods to reduce intraperitoneal pressure, to enhance venous return, and cardiac output, and to improve ventilation [11,13].

\section{Conclusions}

Isolated tension NSP may be an unusual but severe complication of EBUS-TBNA under sedation. All the operators involved in the procedure should know the existence of this condition because of its potentially dramatic clinical evolution. Prompt recognition and peritoneal decompression are essential to improve hemodynamic and respiratory symptoms. Conservative treatment may be a viable option once gastrointestinal perforation has been excluded. In order to reduce the possibility of NSP during EBUS-TBNA, the correct position of the NPC should be endoscopically confirmed before high-flow rate oxygen administration. Moreover, in case of respiratory failure during the procedure requiring invasive ventilatory support, parameters of mechanical ventilation should always be carefully controlled.

\section{References}

1. Wahidi MM, Herth F, Yasufuku K, et al. Technical aspects of endobronchial ultrasound-guided transbronchial needle aspiration. CHEST guideline and expert panel report. Chest 2016;149:816-35.

2. Agostini L, Facciolongo N, Lusuardi M, et al. Endobronchial ultrasound-guided transbronchial needle aspiration under conscious sedation with meperidine and midazolam. Monaldi Arch Chest Dis 2017;87:768.

3. Conte SC, Spagnol G, Confalonieri M, Brizi B. Deep sedation versus minimal sedation during endobronchial ultrasound transbronchial needle aspiration. Monaldi Arch Chest Dis 2018;88:967.

4. Çağlayan B, Yılmaz A, Bilaçeroğlu S, et al. Complications of convexprobe endobronchial ultrasound-guided transbronchial needle aspiration: a multi-center retrospective study. Respir Care 2016;61:243-8.

5. Mularski RA, Sippel JM, Osborne ML. Pneumoperitoneum: a review of nonsurgical causes. Crit Care Med 2000;28:2638-44.

6. Yserbyt J, De Maeyer N, Dooms C, et al. The feasibility of tracheal oxygen supplementation during flexible bronchoscopy. Respiration 2016;92:48-52.

7. Chhajed PN, Aboyoun C, Malouf MA, et al. Management of acute hypoxemia during flexible bronchoscopy with insertion of a nasopharyngeal tube in lung transplant recipients. Chest 2002;121:1350-4.

8. Giménez Velando A, Rodriguez Nieto MJ, Flandes Aldeyturriaga J. Gastric perforation during bronchoscopy for the delivery of oxygen therapy by nasopharyngeal cannula. Arch Bronconeumol 2018;54:284-96. 
9. Ono Y, Kakamu T, Kikuchi H, et al. Expert-performed endotracheal intubation-related complications in trauma patients: incidence, possible risk factors, and outcomes in the prehospital setting and emergency department. Emerg Med Int 2017;10:28.

10. Okamoto A, Nakao A, Matsuda K, et al. Non-surgical pneumoperitoneum associated with mechanical ventilation. Acute Med Surg 2014;1:254-5.
11. Ahmed K, Amine EG, Abdelbaki A, et al. Airway management: induced tension pneumoperitoneum. Pan Afr Med J 2016;25:125.

12. Yoo JH, Shin SJ, Lee KM, et al. Risk factors for perforations associated with endoscopic submucosal dissection in gastric lesions: emphasis on perforation type. Surg Endosc 2012;26:2456-64.

13. Khan ZAJ, Novell JR. Conservative management of tension pneumoperitoneum. Ann R Coll Surg Engl 2002;84:164-5. 